\title{
La actitud hacia el trabajo en equipo en estudiantes universitarios
}

\author{
María Yolanda González Alonso ${ }^{\mathrm{a}}$, Mónica Cano Rosás ${ }^{\mathrm{b}}$, Estíbaliz Jiménez Arberás ${ }^{\mathrm{c}}$ y \\ Thais Pousada García ${ }^{\text {. }}$ \\ ${ }^{\mathrm{a}}$ Universidad de Burgos, ${ }^{\mathrm{b}}$ Universidad de Salamanca, ${ }^{\mathrm{c}}$ Universidad de Oviedo, ${ }^{\mathrm{d}}$ Universidad de la \\ Coruña.
}

\begin{abstract}
Teamwork is considered a valuable competence in university teaching to acquire interpersonal and cognitive skills. The main objective is to analyze the perspectives and experiences that each student has had in relation to the groups to guide the active methodology that will be applied in the teachinglearning process of the subject. It is a quantitative, descriptive, interpretative investigation. The students of the first grade level $(n=69)$ answer a questionnaire about group attitudes at the beginning of the subject. The results confirm the importance of teamwork for students, the perception of their attitude towards teamwork is good, although only $55.1 \%$ is more inclined towards positive attitudes towards teams. The data provided help in the design of an innovation experience by applying an active methodology in which knowledge about positive attitudes to work in a team and apply them in different tasks must be included. The acquisition of these competences must be continuous and progressive on the part of the student and requires specific feedback on the correct behaviors to work effectively as a team and an evaluation that accompanies the learning process.
\end{abstract}

Keywords: Interaction, Cooperative learning, Evaluation, Interdependence, Responsibility, Feedback.

\section{Resumen}

El trabajo en equipo se considera una competencia valiosa en la enseñanza universitaria para adquirir destrezas interpersonales y cognitivas. El objetivo es analizar las perspectivas y experiencias que cada estudiante ha tenido en relación con los grupos para orientar la metodología activa que se aplicará en el proceso enseñanza aprendizaje de la asignatura. Se trata de una investigación cuantitativa, descriptiva interpretativa. El alumnado de primer curso de grado $(n=69)$ contesta al principio de la asignatura a un cuestionario sobre actitudes grupales. Los resultados confirman la importancia que tiene el trabajo en equipo para los estudiantes, la percepción sobre su actitud hacia el trabajo en equipo es buena, aunque solo el 55,1\% se inclina más a actitudes positivas ante los equipos. Los datos aportados ayudan en el diseño de una experiencia de innovación aplicando una metodología activa en la que se deben incluir conocimiento sobre cuáles son las actitudes positivas para trabajar en equipo y aplicarlas en las diferentes tareas. La adquisición de estas competencias debe ser continua y progresiva por parte del estudiante y requiere retroalimentación específica 
sobre las correctas conductas para trabajar eficazmente en equipo y una evaluación que acompañe el proceso de aprendizaje.

Palabras clave: Interacción, Aprendizaje cooperativo, Evaluación, Interdependencia, Responsabilidad, Retroalimentación.

\section{Introducción}

La sociedad exige la formación de universitarios en conocimientos actualizados, en habilidades y en competencias específicas sobre su carrera y también se necesita aprender a crecer y progresar en competencias personales y profesionales cambiantes y cada vez más complejas. La transmisión de actitudes, como la capacidad de trabajo en equipo es una de las labores más difíciles (Richardson, Dawson, Sadlo, Jenkins y Mcinnes, 2007).

En las últimas décadas se ha desarrollado una corriente importante en torno al trabajo en equipo y a los beneficios que pueden esperarse en cuanto a la efectividad y mejora de la calidad en todos los ámbitos del trabajo donde se necesita la participación de diversas disciplinas y profesionales, como es el caso de materias de ciencias de la salud (Durante y Noya, 2003).

La relevancia del trabajo en equipo se ha tratado por diferentes autores como herramienta para el desarrollo exitoso de la actividad docente que pretende la adquisición de conocimientos y el desarrollo de habilidades del alumnado (Pegalajar y Colmenero, 2013). Actualmente es frecuente programar diversas actividades donde el estudiante trabaja en grupo, participa en seminarios, elabora proyectos y diseña talleres (González-Alonso y Escolar-Llamazares, 2017).

El grupo ejerce una influencia muy poderosa sobre el individuo, puede modelar y modificar muchos comportamientos, aptitudes y actitudes individuales. En la Universidad se ha justificado su utilidad y validez académica considerándose una competencia muy demandada en el ámbito profesional y laboral, objeto de estudio en innovación docente (Fidalgo, 2016).

\subsection{Aprendizaje basado en competencias}

El concepto competencia ocupa un amplio espacio de reflexión dentro del modelo educativo universitario que puede definirse como el conjunto de conocimientos, actitudes y destrezas necesarias para desempeñar una función (Le Boterf, 2001).

El trabajo en equipo se define como una competencia básica a desarrollar por el estudiante en cualquier titulación, teniendo ésta un carácter transversal a lo largo de su itinerario (Zubimendi, Ruiz, Carrascal, y De la Presa, 2010) y siendo valiosa para la formación integral de los estudiantes.

Entre las estrategias de enseñanza-aprendizaje que el docente debe potenciar en el estudiante se encuentra la competencia del trabajo en equipo. Se trata de que el alumnado sea capaz de colaborar y participar como miembro de un grupo, asumiendo la responsabilidad y el compromiso propios hacia las tareas y funciones que se tienen

(cc) EY-NC-ND 2018, Universitat Politècnica de València

Congreso IN-RED (2018) 
asignadas para la consecución de unos objetivos comunes, siguiendo los procedimientos acordados y atendiendo a los recursos disponibles (Jacques y Jacques, 2007).

El trabajo en equipo también forma parte del perfil competencial del profesorado universitario junto con la competencia interpersonal, la metodológica, la comunicativa, la de planificación, la gestión de la docencia y la competencia de innovación (Zamorano y Parejo, 2016).

$\mathrm{Al}$ revisar la literatura aparecen numerosas investigaciones que argumentan que el trabajo en equipo contribuye a éxitos académicos de los estudiantes y al desarrollo de otras habilidades, estableciendo conexión entre aprendizajes teóricos y prácticos (Fernández, 2006; Nam y Zellner, 2011; Pujolás, 2008). Aunque no todas las experiencias han tenido éxito, Ariza y Oliva (2004) señalan algunos inconvenientes como la pérdida de iniciativa y compromiso, la conformidad, el retraso en el proceso del trabajo, el desentendimiento y los conflictos.

El aprendizaje en el aula debe ir dirigido a desarrollar prácticas didácticas innovadoras, que favorezcan la reflexión, la participación y la colaboración en la construcción de conocimientos. El trabajo en equipo supone un desafío para el profesorado que tiene que desarrollar nuevos enfoques metodológicos, más inductivos, críticos y colaborativos (Cano, 2005). En las metodologías que aplican trabajo en equipo los resultados son positivos, se mejora el clima de trabajo, las actitudes del estudiante y su grado de motivación, así como su rendimiento (Korkmaz, 2012).

El reto es determinar que metodología activa debe utilizar el docente para potenciar las habilidades de trabajo en equipo que son necesarias y así desarrollar esta competencia.

\subsection{El trabajo en equipo}

Diferentes autores han investigado la eficacia del trabajo en equipo en el aula demostrando que esta metodología enseña estrategias y habilidades de cooperación, facilita la comprensión de los aprendizajes, aumenta su rendimiento educativo y fomenta actitudes de respeto, tolerancia e interacción entre los estudiantes (Villa y Poblete, 2007). Aunque esta forma de enseñanza requiere un gran esfuerzo dinamizador e innovador por parte del profesorado.

A través de estos métodos se trata de lograr según Johnson y Johnson (2000) cinco elementos esenciales: uno, interdependencia positiva, cuando el estudiante piensa que está ligado con sus compañeros de manera que no tendrá éxito si los demás miembros del equipo tampoco lo tienen; dos, responsabilidad individual, si cada miembro rinde cuentas no solo de su parte del trabajo, también del trabajo del resto; tres, interacción cara a cara, los estudiantes se ayudan, se asisten, se animan y se apoyan en su esfuerzo por aprender de manera que se reúnan para ello; cuatro, habilidades sociales, tales como liderazgo, capacidades de decisión, de generar confianza y comunicación y de gestión de conflictos; y cinco, procesamiento grupal autónomo, que supone que el propio equipo analiza en qué medida se están logrando los objetivos y la relación de trabajo efectiva entre los participantes. 
Esta competencia se consigue a través de un proceso que conlleva la adquisición de diferentes habilidades, difíciles de alcanzar. El primer paso es determinar si el alumnado conocen los aspectos que caracterizan un trabajo en equipo eficiente y el segundo si el propio estudiante considera que su actitud hacia el trabajo en equipo es buena.

Con esta información el docente podrá elegir las actividades y metodología adecuada, se trata de adaptarlas a los objetivos de enseñanza aprendizaje, a la madurez y entrenamiento de los participantes, al tamaño del grupo, al ambiente físico, a las características de los miembros y a la capacidad del docente (López et al., 2014).

La hipótesis es que los estudiantes de primer curso valoran su actitud general hacia el trabajo en equipo de manera más positiva que la puntuación obtenida en las diferentes habilidades.

\section{Objetivos}

El objetivo de esta investigación es analizar las perspectivas y experiencias que cada estudiante ha tenido en relación con los grupos para orientar la metodología activa que se aplicará en el proceso enseñanza aprendizaje de la asignatura.

Para lo cual se plantea valorar al principio de curso la actitud hacia el trabajo en equipo del alumnado de primero de grado y analizar las diferencias entre la percepción subjetiva de cada estudiante sobre su actitud hacia el trabajo en equipo y el resultado obtenido en el cuestionario. A partir de este análisis se pretende orientar los contenidos y metodología de la asignatura para que al finalizar el curso el alumnado trabaje en equipo de manera eficaz.

Las preguntas claves fueron: ¿qué actitud para trabajar en equipo consideran que tienen los alumnos de primero en la universidad?, ¿coincide la actitud que cada estudiante cree que tiene, con su conocimiento sobre las habilidades que debe tener para trabajar en equipo de manera eficaz?, y ¿cómo influye esto en la planificación de la metodología enseñanzaaprendizaje del docente?

\section{Desarrollo de la innovación}

La experiencia se ha llevado a cabo en una asignatura de Grado de Terapia Ocupacional que se imparte en la Universidad de Burgos, durante el curso 2016-2017.

El grado de terapia ocupacional tiene unas competencias básicas, generales, transversales y específicas que ha de cumplir el alumnado cuando acaba su etapa de formación. Dentro de las competencias generales que se aprenden en esta asignatura está desarrollar la práctica profesional con respecto a otros profesionales, adquiriendo habilidades de trabajo en equipo.

\subsection{Participantes}

En esta investigación se ha utilizado una muestra de conveniencia no probabilística, formada por 69 estudiantes de primer curso del grado de Terapia Ocupacional. El alumnado matriculado en carreras de Ciencias de la Salud, en concreto en Terapia Ocupacional está formado principalmente por mujeres, en este curso el $85,5 \%$ son mujeres y la edad media es de 19 años. Se aplicó en una materia obligatoria, semestral, eminentemente experimental

(cc) EY-NC-ND 2018, Universitat Politècnica de València

Congreso IN-RED (2018) 
donde el tamaño real del grupo secundario está formado por un número reducido de estudiantes (15) y la asignatura cuenta con 6 créditos (ECTS).

\subsection{Instrumento}

Los datos que se incluyen en este estudio se han obtenido de un cuestionario sobre la Actitud ante los grupos, elaborado por Trechera, 2003 (Anexo I).

El cuestionario plantea treinta preguntas sobre situaciones grupales para conocer las distintas perspectivas y experiencias que cada estudiante ha tenido en relación a los equipos de trabajo. Para responder al cuestionario se rellena la puntuación de una escala Likert de 5 opciones donde 1 es totalmente en desacuerdo y 5 es totalmente de acuerdo. Al sumar las elecciones realizadas se obtiene una puntuación numérica que indica hacia dónde se inclina el alumnado en cuanto a dos posturas extremas: postura positiva hacia el trabajo en equipo y la contraria, negativa, que considera el equipo como una pérdida de tiempo. Aunque lo importante no es tanto la puntuación numérica, sino hacia dónde va la tendencia individual y los componentes del equipo, ya que indicará qué actitud subyace respecto al trabajo en equipo.

Al final del cuestionario se realiza una pregunta sobre la valoración subjetiva de cada estudiante, sobre su actitud para trabajar en equipo del 1 al 5 , siendo 1 muy mala y 5 muy buena.

\subsection{Procedimiento}

El primer día de clase de la asignatura Recursos y herramientas de la Terapia Ocupacional, todos los estudiantes asistentes rellenan un cuestionario sobre su actitud ante los grupos. Se les informa sobre los objetivos y se les dan las instrucciones previas para la adecuada cumplimentación, aplicando los criterios éticos y garantizando la confidencialidad de los datos.

\subsection{Tratamiento de datos}

Una vez recogidos los datos, con el programa estadístico SPSS (versión 24) se llevaron a cabo los análisis descriptivos. Se analizan los datos obtenidos tanto con el cuestionario como la valoración subjetiva y se comparan ambas en cada estudiante. Esta información ayuda a identificar en que habilidades se debe insistir más y tenerlo en cuenta en el diseño metodológico de la asignatura.

\section{Resultados}

Han participado en el estudio 69 estudiantes de los 73 matriculados en la asignatura.

Teniendo en cuenta los objetivos de la investigación se realizó en primer lugar un análisis descriptivo de los datos obtenidos en el cuestionario y así se puede observar la media y la desviación típica de los ítems con los que los estudiantes están más en desacuerdo y más de acuerdo. Se comprueba que de las treinta preguntas del cuestionario, en la tabla 1 aparecen las cuatro más elegidas que muestran su actitud más positiva y destaca el ítem si hay buen clima grupal y las personas están a gusto en el equipo, se implicarán y funcionarán mejor. Y de las cuatro que se alejan más de la actitud positiva que señalan más estudiantes (tabla 
2), destaca si los miembros de un equipo están unidos son capaces de conseguir cualquier tipo de objetivo.

Tabla 1. Ítems con actitud más positiva ante el equipo

ITEM

MEDIA

D. TÍPICA

$\begin{array}{lcc}\begin{array}{l}\text { 29. Si hay buen clima grupal y las personas están a gusto en el } \\ \text { equipo, se implicarán y funcionarán mejor }\end{array} & 1,40 & 0,92 \\ \begin{array}{l}\text { 8. Es importante que los miembros de una organización tengan que } \\ \text { conocer los objetivos, medios y actuaciones de la misma }\end{array} & 1,44 & 0,58 \\ \begin{array}{l}\text { 22. Comunicar y saber escuchar es fundamental para un buen } \\ \text { funcionamiento del equipo }\end{array} & 1,46 & 0,99 \\ \begin{array}{l}\text { 25. Es más efectivo que en el equipo las decisiones se tomen por } \\ \text { consenso }\end{array} & 1,91 & 1,08 \\ \end{array}$

Tabla 2. Ítems con actitud más negativa ante el equipo

ITEM

MEDIA

D. TÍPICA

6. Si los miembros de un equipo están unidos son capaces de 4,56 conseguir cualquier tipo de objetivo.

13. Es importante para ser operativos que las normas en un equipo ya estén dadas y sean claras para que no se pierda tiempo en organizarse o tener que elaborarlas

11. Para que el líder de un equipo sea imparcial, es mejor que no se implique afectivamente con los miembros

1. El ser humano tiende al conformismo y a dejarse influir por los demás

Las puntuaciones totales obtenidas por los estudiantes en el cuestionario se encuentran entre 58 y 102, teniendo en cuenta que la puntuación total de la actitud más positiva ante los grupos es 30 y la más negativa es 150. Si se divide la puntuación en 5 grupos: 5 (puntuación entre 30-54), 4 (puntuación entre 55-79), 3 (puntuación entre 80-104), 2 (puntuación entre 105-129) y 1 (puntuación entre 130-150) se puede ver que todos los estudiantes se encuentran entre el grupo 3 y 4 ).

En cuanto a los resultados que se obtienen tras el análisis de las valoraciones respecto a la percepción subjetiva que el estudiante tiene sobre su propia actitud para trabar en equipo, las puntuaciones se encuentran en un rango, entre 2-5 con una media de 4.

Al comparar ambas puntuaciones se comprueba que mientras el rango en el cuestionario está entre 3-4 siendo la puntuación media 3,5. La percepción del alumnado tiene un rango

(cc) EY-NC-ND 2018, Universitat Politècnica de València

Congreso IN-RED (2018) 
más amplio de 2 a 5, y medio punto de diferencia con la media de la percepción subjetiva. En menos de la mitad de los estudiantes coincide la puntuación subjetiva y la puntuación del cuestionario (36,3 \%), más de la mitad se valoran con un punto por encima $(50,7 \%)$ y muy pocos (8,7\%) se valoran por debajo. El 4,3\% se valoran dos puntos o más de diferencia tanto por arriba como por debajo. El mayor porcentaje en ambas puntuaciones está en 4 . La mayoría de los estudiantes dicen estar más cerca de la actitud positiva hacia los equipos $(65,9 \%)$ y en la puntuación en el cuestionario también, aunque más baja (55,1\%) (Tabla 3).

Tabla 3. Porcentajes de puntuación positiva en el cuestionario y percepción subjetiva de la actitud hacia el trabajo en equipo

\section{PUNTUACIÓN PERCEPCIÓN}

RANGO EN EL CUESTIONARIO

DEL ESTUDIANTE

\begin{tabular}{ccccc}
\hline & $\mathbf{\%}$ & $\mathbf{\%}$ & $\mathbf{\%}$ & $\mathbf{N}$ \\
5 & 0 & 0 & 17,4 & 12 \\
4 & $\mathbf{5 5 , 1}$ & 38 & $\mathbf{6 5 , 9}$ & 45 \\
3 & 44,9 & 31 & 15,9 & 11 \\
2 & 0 & 0 & 1,5 & 1 \\
1 & 0 & 0 & 0 & 0 \\
\hline TOTAL & 100 & 100 & 100 & 69 \\
\hline
\end{tabular}

Se puede apreciar que las posturas más negativas podrían impedir un trabajo en equipo eficaz, sin embargo son muchos los estudiantes que consideran que su actitud hacia el trabajo en equipo es muy positiva. Por lo que parece difícil que se preocupen de cambiar algunas de sus actuaciones para trabajar mejor en equipo, puesto que creen hacerlo bien. El ítem que mayor desviación típica presenta, es la pregunta relacionada con el líder para que el líder de un equipo sea imparcial, es mejor que no se implique afectivamente con los miembros.

Estos análisis proporcionan información valiosa a tener en cuenta por el docente en el diseño de metodologías y así potenciar estas habilidades acerca de esta competencia de trabajo en equipo.

\section{Conclusiones}

En definitiva, a pesar de las valoraciones altas en cuanto a la actitud positiva hacia el trabajo en equipo, el profesorado debe considerar algunos aspectos básicos que no tienen adquiridos los estudiantes, por lo que se hace necesario poner en marcha estrategias que faciliten el desarrollo de las habilidades de trabajo en equipo en el aula. El primer paso fue dar a conocer al estudiante que no cuenta con actitudes tan positivas como se cree y que sepa cuáles son sus errores. Para posteriormente conocer y practicar a través de metodologías activas las habilidades que se requieren en un trabajo en equipo para que sea 
eficaz y recibir retroalimentación continua sobre aquellas que se van adquiriendo, bien sea información de los compañeros y/o del docente.

En este caso se deseñaron metodologías activas como el puzle, el seminario, el aprendizaje basado en problemas, juegos de roles, talleres y estudio de casos. El propósito fue mejorar habilidades en estos estudiantes como el diseño de objetivos comunes, la aplicación del consenso de manera eficaz, la gestión de las emociones, el manejo del liderazgo y la reflexión y el análisis de contenidos. Es decir practicar al menos las cuatro habilidades con las que se encontraban más de acuerdo en el cuestionario que, reflejaban actitudes negativas hacia el trabajo en equipo.

Los futuros terapeutas ocupacionales, tendrán que desempeñar su empleo en diferentes grupos y con personas heterogéneas, lo cual convierte el trabajo en equipo en una competencia muy necesaria. Aunque durante su formación los estudiantes realizan diferentes trabajos en grupos, estas actividades por si solas no garantizan que a lo largo de todos los cursos se adquieran las capacidades requeridas si no reciben formación específica y retroalimentación continua. Hay que aprovechar que el alumnado tiene una actitud positiva aunque un primer paso es hacerles comprender que les faltan habilidades o que necesitan mejorarlas.

Una de las limitaciones de este estudio se debe al propio diseño descriptivo transversal que no permite generalizar los datos. Para futuras investigaciones se propone conocer la percepción del alumnado de otros cursos y de otras Universidades y valorar al estudiante al principio y al final de la impartición de la asignatura para comprobar si se adquiere una actitud más positiva hacia el trabajo en equipo tras aplicar las metodologías activas más apropiadas. No hay que olvidar, que el docente debe proporcionar una retroalimentación continua mientras el estudiante utiliza cada una de las técnicas. Así se va consiguiendo el cambio en aquellas actitudes que están frenando la adquisición de habilidades para un trabajo en equipo eficaz.

\section{Referencias}

ARIZA, A. Y OLIVA, S. (2004). "Las nuevas tecnologías de la información y la comunicación y una propuesta de trabajo colaborativo”. Comunidad Virtual de Tecnología y Liderazgo.

CANO, E. (2005). Cómo mejorar las competencias docentes. Guía para la autoevaluación y el desarrollo de competencias del profesorado. Barcelona. Graó.

DURANTE, P. Y NOYA, B. (2003). “Trabajo en equipo. Contribución del terapeuta ocupacional”. En Polonio, B., Durante, P. y Noya, B. Conceptos fundamentales de terapia ocupacional. Madrid: Panamericana.

FERNÁNDEZ, M. (2006). "Metodologías activas para la formación de competencias”. Education silo XXI, 24, 35-56.

FIDALGO, Á. (2016). “La innovación docente y los estudiantes”. La cuestión universitaria, (7), 8491.

(cc) BY-NC-ND 2018, Universitat Politècnica de València

Congreso IN-RED (2018) 
GONZÁLEZ-ALONSO, MY. Y ESCOLAR-LLAMAZARES, MC. (2017). "Valoración de las estrategias de participación del alumnado en el aula universitaria". Actas de las IX Jornadas de Redes de Investigación en Innovación Docente de la UNED. Madrid, junio 2017.

JACQUES, J. Y JACQUES, P. (2007). Cómo trabajar en equipo: Guía práctica. Madrid: Narcea Ediciones

JOHNSON, D. W Y JOHNSON, R. T. (2000). Cooperative learning. Buenos Aires. Paidos.

KORKMAZ, Ö. (2012). “A validity and reliability study of Online Cooperative Learning Attitude Scale (OCLAS)”. Computers \& Education, 59, 1162-1169.

LE BOTERF, G. (2001). Ingeniería de las competencias. Barcelona. Gedes

LÓPEZ, B. G., BERTOMEU, I. M., CHORNET, S. I., OLMEDO, M. J. C., OLTRA, S. N., Y FÉLIX, E. G. (2014). “Metodología centrada en el aprendizaje. Su impacto en las estrategias de aprendizaje y en el rendimiento académico de los estudiantes universitarios”. Revista española de pedagogía, 415-435.

NAM, CW. Y ZELLNER, RD. (2011). "The relative effects of positive interdependence and group processing on student achievement and attitude in online cooperative learning”. Computers \& Education, 56, 680-688.

PEGALAJAR, MC. Y COLMENERO, MJ. (2013). "Percepciones hacia el aprendizaje cooperativo en estudiantes del Grado de Maestro”. Revista Docencia Universitaria. REDU. Vol. 11 (3).

PUJOLÁS, P. (2008). El aprendizaje cooperativo: 9 ideas clave. Barcelona: Graó.

RICHARDSON, J. T., DAWSON, L., SADLO, G., JENKINS, V., Y MCINNES, J. (2007). "Perceived academic quality and approaches to studying in the health professions". Medical Teacher, 29 (5), e108-e116.

TRECHERA, J. L. (2003). Trabajar en Equipo: Talento y talante. Técnicas de dinámica de grupos. Bilbao: Desclée de Brouwer, S. A.

VILLA, A. Y POBLETE, M. (2007). Aprendizaje Basado en Competencias. Una propuesta para la evaluación de Competencias genéricas. Bilbao: Mensajero.

ZABALZA, M. Á. (2016). “Ser profesor universitario hoy”. La cuestión universitaria, (5), 68-80.

ZAMORANO, S., Y PAREJO, J. L. (2016). "La renovación de las metodologías educativas como garantía de calidad institucional”. La Cuestión Universitaria, (2), 45-64.

ZUBIMENDI, JL., RUIZ, MP, CARRASCAL, E. Y DE LA PRESA, H. (2010). El aprendizaje cooperativo en el aula universitaria. Manual de ayuda al profesorado. Universidad del País Vasco. 
Nombre y apellidos:

Fecha de nacimiento:

Fecha de hoy:

\section{TRABAJO EN EQUIPO}

Conteste a cada una de las proposiciones que aparecen a continuación según su grado de acuerdo o desacuerdo.

\begin{tabular}{|c|c|c|c|c|}
\hline $\begin{array}{c}\text { Totalmente en } \\
\text { desacuerdo }\end{array}$ & $\begin{array}{c}\text { En } \\
\text { desacuerdo }\end{array}$ & $\begin{array}{c}\text { Tengo } \\
\text { dudas }\end{array}$ & $\begin{array}{c}\text { De } \\
\text { acuerdo }\end{array}$ & $\begin{array}{c}\text { Totalmente de } \\
\text { acuerdo }\end{array}$ \\
\hline 1 & 2 & 3 & 4 & 5 \\
\hline
\end{tabular}

\begin{tabular}{|c|l|c|c|c|c|c|}
\hline 1 & $\begin{array}{l}\text { El ser humano tiende al conformismo y a dejarse } \\
\text { influir por los demás }\end{array}$ & 1 & 2 & 3 & 4 & 5 \\
\hline 2 & Es más rentable trabajar solola que en un equipo & 1 & 2 & 3 & 4 & 5 \\
\hline 3 & $\begin{array}{l}\text { Para ser operativos en la toma de decisiones, hay } \\
\text { que imponer decisiones por mayoria }\end{array}$ & 1 & 2 & 3 & 4 & 5 \\
\hline 4 & $\begin{array}{l}\text { El ideal de una organización es que todo el mundo } \\
\text { piense sienta y actúe de la misma manera }\end{array}$ & 1 & 2 & 3 & 4 & 5 \\
\hline 5 & $\begin{array}{l}\text { Si hay conficto en el equipo, lo más efectivo es } \\
\text { expulsar a los miembros discordantes }\end{array}$ & 1 & 2 & 3 & 4 & 5 \\
\hline 6 & $\begin{array}{l}\text { Si los miembros de un equipo están unidos son } \\
\text { capaces de conseguir cualquier tipo de objetivo }\end{array}$ & 1 & 2 & 3 & 4 & 5 \\
\hline 7 & $\begin{array}{l}\text { En general, en los equipos, los miembros se } \\
\text { esfuerzan menos que individualmente }\end{array}$ & 1 & 2 & 3 & 4 & 5 \\
\hline 8 & $\begin{array}{l}\text { No es importante que los miembros de una } \\
\text { organización tengan que conocer los objetivos, } \\
\text { medios y actuaciones de la misma }\end{array}$ & 1 & 2 & 3 & 4 & 5 \\
\hline 9 & $\begin{array}{l}\text { En los equipos es más fácil presionar a los } \\
\text { miembros para que unos pocos se salgan con la } \\
\text { suya }\end{array}$ & 1 & 2 & 3 & 4 & 5 \\
\hline 10 & $\begin{array}{l}\text { Para mantener la identidad de un equipo, es } \\
\text { preferible que se aisle de las influencias extemas }\end{array}$ & 1 & 2 & 3 & 4 & 5 \\
\hline 11 & $\begin{array}{l}\text { Para que el lider de un equipo sea imparcial, es } \\
\text { mejor que no se implique afectivamente con los } \\
\text { miembros }\end{array}$ & 1 & 2 & 3 & 4 & 5 \\
\hline 12 & $\begin{array}{l}\text { Es preferible que los miembros sean homogéneos } \\
\text { en conocimiento, formación, experiencia, actitudes, } \\
\text { etc. }\end{array}$ & 1 & 2 & 3 & 4 & 5 \\
\hline 13 & $\begin{array}{l}\text { Es importante para ser operativos que las normas } \\
\text { en un equipo ya estén dadas y sean claras para que } \\
\text { no se pierda tiempo en organizarse o tener que } \\
\text { elaborarlas }\end{array}$ & 1 & 2 & 3 & 4 & 5 \\
\hline
\end{tabular}

1

(cc) EY-NC-ND 2018, Universitat Politècnica de València Congreso IN-RED (2018) 


\begin{tabular}{|c|c|c|c|c|c|c|}
\hline 14 & $\begin{array}{l}\text { En un equipo siempre los intereses individuales } \\
\text { tiene que estar supeditados a los del equipo }\end{array}$ & 1 & 2 & 3 & 4 & 5 \\
\hline 15 & $\begin{array}{l}\text { Muchas personas no descubren sus capacidades } \\
\text { hasta que no forman parte de un equipo }\end{array}$ & 1 & 2 & 3 & 4 & 5 \\
\hline 16 & $\begin{array}{l}\text { Para tomar decisiones rápidas y evitar conflictos, es } \\
\text { preferible un liderazgo autoritario }\end{array}$ & 1 & 2 & 3 & 4 & 5 \\
\hline 17 & $\begin{array}{l}\text { Lo fundamental para un equipo es ser efectivo y } \\
\text { cumplir los objetivos que se ha planteado }\end{array}$ & 1 & 2 & 3 & 4 & 5 \\
\hline 18 & El trabajo en equipo es más lento y menos efectivo & 1 & 2 & 3 & 4 & 5 \\
\hline 19 & $\begin{array}{l}\text { Los equipos deben formarse y evolucionar } \\
\text { espontáneamente }\end{array}$ & 1 & 2 & 3 & 4 & 5 \\
\hline 20 & $\begin{array}{l}\text { Es imposible comunicar y poder llegar a acuerdos } \\
\text { con personas que piensan de forma distinta }\end{array}$ & 1 & 2 & 3 & 4 & 5 \\
\hline 21 & $\begin{array}{l}\text { Los equipos "Uniformizan" a sus integrantes; "Dime } \\
\text { con quién andas y te diré quién eres" }\end{array}$ & 1 & 2 & 3 & 4 & 5 \\
\hline 22 & $\begin{array}{l}\text { Comunicar y saber escuchar es fundamental para } \\
\text { un buen funcionamiento del equipo }\end{array}$ & 1 & 2 & 3 & 4 & 5 \\
\hline 23 & $\begin{array}{l}\text { La capacidad para trabajar en equipo es innata al } \\
\text { sujeto }\end{array}$ & 1 & 2 & 3 & 4 & 5 \\
\hline 24 & $\begin{array}{l}\text { El equipo puede hacer que los integrantes se } \\
\text { esfuercen menos individualmente o se dejen llevar }\end{array}$ & 1 & 2 & 3 & 4 & 5 \\
\hline 25 & $\begin{array}{l}\text { Es más efectivo que en el equipo las decisiones se } \\
\text { tomen por consenso }\end{array}$ & 1 & 2 & 3 & 4 & 5 \\
\hline 28 & $\begin{array}{l}\text { Tener información es tener poder, de ahí que para } \\
\text { dirigir bien un equipo no haya que comunicar las } \\
\text { cosas }\end{array}$ & 1 & 2 & 3 & 4 & 5 \\
\hline 27 & $\begin{array}{l}\text { Es más efectivo trabajar como diez que hacer } \\
\text { trabajar a diez }\end{array}$ & 1 & 2 & 3 & 4 & 5 \\
\hline 28 & $\begin{array}{l}\text { "Más vale lo malo conocido que lo bueno por } \\
\text { conocer", es preferible mantener las actividades que } \\
\text { ya se dominan que abrirse a nuevos experimentos }\end{array}$ & 1 & 2 & 3 & 4 & 5 \\
\hline 29 & $\begin{array}{l}\text { Si hay buen clima grupal y las personas están a } \\
\text { gusto en el equipo se implicarán y funcionará mejor }\end{array}$ & 1 & 2 & 3 & 4 & 5 \\
\hline 30 & $\begin{array}{l}\text { Para no perder tiempo en el trabajo en equipo, es } \\
\text { más efectivo un liderazgo directivo }\end{array}$ & 1 & 2 & 3 & 4 & 5 \\
\hline
\end{tabular}

¿Cuál consideras que es tu actitud para trabajar en equipo del 1 al 5 , siendo 1 una actitud mury mala y 5 muy buena? 